\title{
Efficacy of bilateral lower limb training over unilateral to re-educate balance and walking in post stroke survivors: a protocol for randomized clinical trial
}

\section{Pallavi Harjpal}

Ravi Nair Physiotherapy College, Datta Meghe Institute of Medical Sciences https://orcid.org/00000002-3829-7650

Dr, Mohd Irshad Qureshi ( $\square$ irshadphysio@rediffmail.com )

Ravi Nair Physiotherapy College, Datta Meghe Institute of Medical Sciences https://orcid.org/00000002-9379-4879

\section{Rakesh Krishna Kovela}

Ravi Nair Physiotherapy College, Datta Meghe Institute of Medical Sciences https://orcid.org/00000002-3744-4778

\section{Method Article}

Keywords: Bilateral training, Unilateral training, Lower Limb, Balance, Walking, Non-paretic side Stroke, Rehabilitation.

Posted Date: May 28th, 2021

DOl: https://doi.org/10.21203/rs.3.pex-1497/v1

License: (9) (1) This work is licensed under a Creative Commons Attribution 4.0 International License. Read Full License

Version of Record: A version of this preprint was published at Journal of Pharmaceutical Research International on December 13th, 2021. See the published version at https://doi.org/10.9734/jpri/2021/v33i56A33912. 


\section{Abstract}

Background: Stroke is the leading cause of global morbidity and a major contributor to disability. According to the National Stroke Association, as many as 9 out of 10 stroke survivors have some degree of paralysis immediately after the stroke. Aim is to evaluate the level to which the bilateral lower limb training compared to unilateral alone, reinforces the lower limb functional recovery in the subjects with sub-acute stroke.

Methods: The participants $(n=40)$ with subacute stroke will be included in assessor blinded, randomized clinical trial. Participants will be categorized into 2 groups after performing baseline assessments and equal randomization. The participants in Group A will receive only training to the affected side, and those in the Group B will receive bilateral training. We will be evaluating the improvement in lower limb function by balance and walking along with gait parameters.

Discussion: Findings of the study will provide further insight into the effectiveness of bilateral training over unilateral in post stroke survivors. If this study is proved to be effective it will improve the balance and gait impairments in post stroke patients.

The clinical trial registry-India(CTRI) registration number for this trial is CTRI/2021/05/033621.

\section{Introduction}

Stroke as defined by World Health Organization (WHO) "rapidly developing clinical signs of focal (or global) cerebral disorder, with symptoms lasting 24 hours or longer or leading to death, with no apparent cause other than vascular origin"(1).The prevalence rates range from 84 to 262 strokes per 100,000 people in rural areas and in urban areas, between 334 and 424 strokes per 100,000 inhabitants(2). Impaired muscle strength is the major factor of reduced motor function, activity limitation and disability which is seen in 80 to $90 \%$ of post stroke survivors(3). Deficit in the motor function is characterized by hemiplegia (paralysis) or hemiparesis (weakness) on the contralateral side of lesion. Besides that, there are evidences stating presence of mild weakness on the ipsilateral "supposed to be normal" side of lesion $(4,5)$. This can be due to fact that only $75-90 \%$ of corticospinal fiber cross in the medulla to the contralateral side. The remaining fibers are transmitted to the spinal cord ipsilaterally in the ventral or anterior corticospinal tract. Some of these fibers cross once in the spinal cord while the others remain uncrossed, thereby illustrating the cause of bilateral weakness(6). The amount of weakness experienced by the patient may also vary according to the level of inactivity (disuse atrophy) and the specific functional tasks attempted. 
There is enough corroboration that motor functioning is impaired on the unaffected side of post stroke survivors, as indicated through muscle weakness, atrophy or disuse $(7,8)$. Integration of a bilateral training sequentially along with unilateral may be more effective in promoting improvements in hand function in chronic post stroke patients with paresis than unilateral task-oriented training alone (9). In post-stroke survivors, bilateral arm training with auditory cueing has a beneficial effect on functional and motor execution of the affected extremity(10). Post-stroke survivors may benefit from bilateral lower limb strengthening services that boost their ability to get out of a chair (11), climbing stairs, walking and improving the quality of life(12). The intent of this study is to examine the effect of bilateral lower limb training over the unilateral to re-educate balance and walking in post stroke survivors. Here, the taskoriented approach i.e., Motor Relearning Programme and PNF for the affected side along with strength training to the less affected side in bilateral training patients.

"Rehabilitation to the affected side involves relearning of real-life activities" which is done with the help of Motor Relearning Programme. "The actions to be learned are done in a suitable setting, with exercises targeted directly at the muscles and muscle synergies needed for the action to be performed." Analysis of the missing component is done and the practice of that component leading to re-education of motor task. Task specific training is integrated into the therapy session as functional improvement is the major goal of rehabilitation. While most recovery focuses on the affected side, it appears that the less-affected side often requires attention in order to improve equilibrium.

In most of the interventions the affected side is targeted for early rehabilitation while the unaffected side remains unused and loses its competencies. This opens a window for bilateral training over unilateral training to improve the motor function and thereby fasten the recovery process and also help in further rehabilitation once the patient follows the verbal command. This paves a path to design a specific protocol quantifying the need to regain motor control in the affected side as well as to maintain the integrity of unaffected side. So, this study focuses on bilateral training over unilateral on motor control of post stroke survivors.

\section{Reagents}

\section{Equipment}

- Measuring tape

- Knee hammer 
Stop watch

\section{Weight cuff}

Dumbbell

Sensory examination kit (Cotton, All pin etc)

Low couch of convenient height (for standing up and sitting down)

Several small steps

Common objects for retaining hand functions

\section{Procedure}

1. Recruit Subjects $(\mathrm{N}=40)$ : Subjects will be screened by inclusion and exclusion criteria, informed consent \& medical history will be obtained from subjects.

2. Perform baseline assessment.

3. Allocation- Group A (20 subjects), Group B (20 subjects)

4. Group A : 6 weeks of intervention, Affected lower limb training $=20$ mins/day, 5 days per week for 6 weeks.

5. Group B : 6 weeks of intervention, Bilateral lower limb training $=40$ mins/day, 5 days per week for 6 weeks.

6. Perform a post-treatment assessment.

7. Statistical Analysis

\section{Troubleshooting}

\section{Time Taken}

1 year

\section{References}


1. World Health Organization (WHO) Definition of Stroke - Public Health [Internet]. [cited 2021 Jan 25]. Available from: https://www.publichealth.com.ng/world-health-organization-who-definition-of-stroke/

2. Recommendations for the Early Management of Acute Ischemic Stroke: A Consensus Statement for Healthcare Professionals from the Indian Stroke Association - Dheeraj Khurana, M. Vasantha Padma, Rohit Bhatia, Subhash Kaul, Jeyaraj Pandian, P. N. Sylaja, , Deepak Arjundas, Ashok Uppal, V. G. Pradeep, Vinit Suri, D. Nagaraja, Anand Alurkar, Sunil Narayan, , 2018 [Internet]. [cited 2021 Jan 25]. Available from: https://journals.sagepub.com/doi/abs/10.1177/2516608518777935

3. "Physical Rehabilitation, 6th edition” by Susan B. O'Sullivan, Thomas J. Schmitz et al. [Internet]. [cited 2021 Jan 25]. Available from: https://hsrc.himmelfarb.gwu.edu/books/85/

4. Carin-Levy G, Greig C, Young A, Lewis S, Hannan J, Mead G. Longitudinal Changes in Muscle Strength and Mass after Acute Stroke. Cerebrovasc Dis. 2006;21(3):201-7.

5. Adams RW, Gandevia SC, Skuse NF. THE DISTRIBUTION OF MUSCLE WEAKNESS IN UPPER MOTONEURON LESIONS AFFECTING THE LOWER LIMB. Brain. 1990 Oct 1;113(5):1459-76.

6. Robert A. Davidoff MD. The pyramidal tract. Neurology. 1990 Feb 1;40(2):332-332.

7. Bohannon RW, Andrews AW. Limb Muscle Strength is Impaired Bilaterally after Stroke. J Phys Ther Sci. 1995;7(1):1-7.

8. Post-stroke hemiplegia; crural muscle strength and structure - PubMed [Internet]. [cited 2021 Jan 25]. Available from: https://pubmed.ncbi.nlm.nih.gov/6932731/

9. McCombe Waller S, Whitall J, Jenkins T, Magder LS, Hanley DF, Goldberg A, et al. Sequencing bilateral and unilateral task-oriented training versus task oriented training alone to improve arm function in individuals with chronic stroke. BMC Neurol [Internet]. 2014 Dec 14 [cited 2021 Jan 22];14. Available from: https://www.ncbi.nlm.nih.gov/pmc/articles/PMC4276071/

10. Repetitive bilateral arm training with rhythmic auditory cueing improves motor function in chronic hemiparetic stroke - PubMed [Internet]. [cited 2021 Jan 25]. Available from: https://pubmed.ncbi.nlm.nih.gov/11022069/

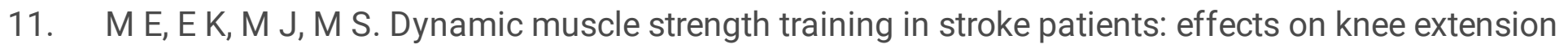
torque, electromyographic activity, and motor function [Internet]. Vol. 76, Archives of physical medicine and rehabilitation. Arch Phys Med Rehabil; 1995 [cited 2021 Jan 25]. Available from:

https://pubmed.ncbi.nlm.nih.gov/7741611/?dopt=Abstract

12. Teixeira-Salmela LF, Olney SJ, Nadeau S, Brouwer B. Muscle strengthening and physical conditioning to reduce impairment and disability in chronic stroke survivors. Arch Phys Med Rehabil. 1999 Oct;80(10):1211-8. 
13. Kanase S. Effect of Motor Relearning Programme and Conventional Training on Functional Mobility in Post Stroke Patients. 2020 May 19;

14. Wu J, Cheng $\mathrm{H}$, Zhang J, Bai Z, Cai S. The modulatory effects of bilateral arm training (BAT) on the brain in stroke patients: a systematic review. Neurol Sci Off $\mathrm{J}$ Ital Neurol Soc Ital Soc Clin Neurophysiol. 2020 Nov 12;

15. Chen P, Kwong PWH, Lai CKY, Ng SSM. Comparison of bilateral and unilateral upper limb training in people with stroke: A systematic review and meta-analysis. PLoS ONE [Internet]. 2019 May 23 [cited 2021 Jan 22];14(5). Available from: https://www.ncbi.nlm.nih.gov/pmc/articles/PMC6532847/

16. IJPR.2019.141 - International Journal of Physiotherapy and Research [Internet]. [cited 2021 Feb 26]. Available from: https://www.ijmhr.org/IntJPhysiotherRes/ijpr-2019-141

17. Jeon HJ, Hwang BY. Effect of bilateral lower limb strengthening exercise on balance and walking in hemiparetic patients after stroke: a randomized controlled trial. J Phys Ther Sci. 2018 Feb;30(2):277-81.

18. Kamalakannan S, Gudlavalleti ASV, Gudlavalleti VSM, Goenka S, Kuper H. Incidence \& prevalence of stroke in India: A systematic review. Indian J Med Res. 2017 Feb 1;146(2):175.

19. Banerjee TK, Das SK. Fifty years of stroke researches in India. Ann Indian Acad Neurol. 2016 Jan 1;19(1):1.

20. Wist S, Clivaz J, Sattelmayer M. Muscle strengthening for hemiparesis after stroke: A metaanalysis. Ann Phys Rehabil Med. 2016 Apr;59(2):114-24.

21. Johannsen L, Wing AM, Pelton T, Kitaka K, Zietz D, Brittle N, et al. Seated Bilateral Leg Exercise Effects on Hemiparetic Lower Extremity Function in Chronic Stroke. Neurorehabil Neural Repair. 2010 Mar 1;24(3):243-53.

22. Jonsdottir J, Cattaneo D. Reliability and Validity of the Dynamic Gait Index in Persons With Chronic Stroke. Arch Phys Med Rehabil. 2007 Nov 1;88(11):1410-5.

23. Mudie MH, Matyas TA. Can simultaneous bilateral movement involve the undamaged hemisphere in reconstruction of neural networks damaged by stroke? Disabil Rehabil. 2000 Jan 1;22(1-2):23-37.

24. Janet H. Carr, Roberta B. Shepherd -Stroke Rehabilitation - Guidelines for Exercise and Training to Optimize Motor Skill 2002 | Evidence Based Medicine | Neuroplasticity [Internet]. [cited 2021 Jan 26]. Available from: https://www.scribd.com/doc/178169431/Janet-H-Carr-Roberta-B-Shepherd-StrokeRehabilitation-Guidelines-for-Exercise-and-Training-to-Optimize-Motor-Skill-2002

25. Todd JS, Shurley JP, Todd TC. Thomas L. DeLorme and the Science of Progressive Resistance Exercise. J Strength Cond Res. 26(11):2913-23. 
Page 7/7 